\title{
Sur une évolution atypique de la peste équine particulière à l'A.O.F.
}

\author{
par P. MORNET
}

LA peste équine sévit à Dakar presque chaque année avec plus ou moins d'intensité, suivant qu'elle est alimentée par des chevaux ou mulets importés, très sensibles, ou des animaux indigènes, plus résistants.

Au cours de l'épidémie de 1942, qui débute en Mars, les seuls animaux atteints sont des animaux indigènes. Aucun cheval (arabe ou barbe) ou mulet, appartenant à l'Administration militaire, seul détenteur à cette époque de sujets importés, ne contracte la maladie.

Première particularité d'une épidémie a forme symptomatologique inaccoutumée.

Sans prodromes, en pleine saison sèche et froide, l'affection éclate le 3 Mars et se manifeste par la mort presque foudroyante de deux chevaux appartenant au Cercle de l'Etrier.

L'autopsie révèle une congestion généralisée : les vaisseaux sont gorgés d'un sang noir et poisseux, la conjonctive et la muqueuse buccale sont jaunes safran, le foie couleur feuille morte, la vessie pleine d'une urine hémoglobinurique.

Les prélèvements en vue d'examens bactériologiques' et parasitaires permettent d'éliminer la coexistence d'une maladie infectieuse à virus figuré ou d'une hématozoose.

Il s'agit bien de peste équine, connue en A.O.F. et à Dakar depuis de longues années, évoluant sous la forme suraiguë.

Sur un effectif de douze chevaux, seuls ces deux cas sont relevés.

C'est alors qu'un nouveau foyer apparait aussi brutalement, le 20 Mars, au Ranch de l'Eperon (Etablissement situé a 2 kilomètres du précédent, qui loue des chevaux aux amateurs d'équitation), foyer dont l'évolution mérite d'être relatée.

La maladie atteint d'abord les chevaux $n^{\circ s} 1$ et 2 , venus huit jours auparavant du village de Malika, situé à une quinzaine de kilomètres de Dakar. Le premier est touché le 20 Mars 1942, le second, le 21. Ils meurent tous les deux dans les 24 heures, après que la propriétaire du Ranch ait constaté, en dehors des signes généraux d'inappétence et d'abattement (stupeur), de l'hémoglobinurie.

La maladie gagne en intensité et, sur les 70 chevaux de l'effectif, 32 sont atteints, dont 11 morts et 21 guéris.

Le tableau ci-dessous (voir page suivante) condense le bilan de l'épizootie.

De ce tableau il découle diverses constatations :

lo l'âge des animaux n'a d'influence ni sur la réceptivité des sujets, ni sur l'évolution de la maladie;

$2^{\circ} l^{\prime}$ affection évolue de façon régulière : sévère au début, elle décroît en malignité assez rapidement. A partir du 3 Avril en effet, la plupart des animaux guérissent;

$3^{\circ}$ la température ne dépasse jamais la normale. Bien plus, certains sujets ne dépassent pas $3605 \mathrm{C}$. Ils sont « froids 》 d'emblée;

$4^{\circ}$ outre cette absence de fièvre, on note fréquemment de l'hémoglobinurie ( 7 cas), des accès nerveux (5 cas), unc odeur fétide caractéristique qui semble s'exhaler du corps de l'animal et surtout des naseaux (6 cas);

$5^{\circ}$ les symptômes cardinaux de la peste equine : inappétence, stupeur, congestion des muqueuses safranées, accélération des grandes fonctions, existent, quoique la présence d'œdèmes n'est point relevée.

Au cours des autopsies pratiquées, des lésions à peu près constantes sont notées : sérosité nasale souvent teintée d'hémoglobine; ictère intense apparaissant dès l'incision cutanée; fortes arborisations vasculaires du conjonctif sous-cutané; congestion généralisée; sang noir et poisseux; poumon congestionné, de même la nappe épiplö̈que; rate hypertrophiée, souvent congestive; foie généralement hypertrophié, cirrhotique, dégénéré; muscles infiltrés, jaunâtres. La vessie, souvent congestionnée, récèle une urine foncée, parfois hémoglobinurique.

Dans plusieurs cas, l'encéphale est congestionné; il y a infiltration sous-arachnoïdienne et de la sérosité dans les ventricules latéraux. 
BILAN DE L'ENZOOTIE DU RANCH DE L'ÉPERON

\begin{tabular}{|c|c|c|c|c|}
\hline $\begin{array}{l}\text { NUMÉRO } \\
\text { du cheval }\end{array}$ & AGE & $\begin{array}{c}\text { DUREEE } \\
\text { de la maIadie }\end{array}$ & SYMPTOMES CARDINAUX & $\begin{array}{r}\text { TERMI- } \\
\text { NAISON }\end{array}$ \\
\hline 1 & 6 ans & 1 jour & Inappétence, tristesse, abattement, hémoglobinurie, pas de fièvre. & Mort \\
\hline 2 & 4 ans & 1 jour & $-d^{0}-$ & Mórt \\
\hline 3 & 6 ans & 10 jours & $\begin{array}{l}\text { Inappétence, tristesse, abattement, muqueuses safranées, avec pété- } \\
\text { chies, T. 3705, violents accès nerveux. }\end{array}$ & Morat \\
\hline 4. & $\mid 3$ a. $1 / 2 \mid$ & 10 jours & - do - & Guéri \\
\hline 5 & $\begin{array}{c}10 \text { ans } \\
\ddots \\
i\end{array} \mid$ & 20 jours & $\begin{array}{l}\text { Inappétence, tristesse, abattement, muqueuses conjonctivale et bućcale } \\
\text { congestionnées, safranées. Violent's accès nerveux : bondit, se jette } \\
\text { contre les parois du box ou sur sa ration puis s'arrête pantelant. } \\
\text { Áccès plus fréquents la nuit que le jour. A partir du } 8 \text { e jour de la ma- } \\
\text { ladie, accès espacés, apparition de troubles oculaires, l'animal semble } \\
\text { aveugle. Le } 20^{e} \text { jour, entre en convalescence mais reste peureux pen- } \\
\text { dant un certain temps. Pas de fièvre. }\end{array}$ & $\begin{array}{l}\vdots \\
\vdots \\
\vdots \\
\vdots\end{array}$ \\
\hline 6 & 4 ans & 4 jours & $\begin{array}{l}\text { Anorexie, tristesse, abattement, conjonctive rouge safran, hémoglo- } \\
\text { binurie, pas de fièvre, odeur fétide exhalée par l'animal." }\end{array}$ & Mort \\
\hline 7 & 6 ans & 3 jours & Mêmes symptômes plus hémoglobinurie. & Mort \\
\hline 8 & 8 ans & 2 jours & $-d^{0}-$ & Mort \\
\hline 9 & 5 ans & 14 jours & - do - & Mort \\
\hline 10 & 4 ans & 3'jours & $-\mathrm{d}^{\mathbf{o}}-\mathrm{d}^{\prime}$ & Mort - \\
\hline 11 & 7 ans & 1 jour & 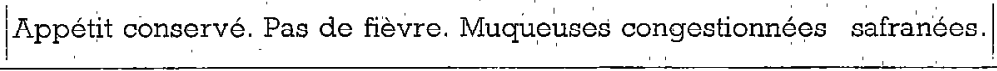 & Mort \\
\hline 12 & 6 ans & 4 jours & $\begin{array}{l}\text { Inappétence, tristesse, abattement, pas de fièvre, muqueuses safranées } \\
\text { avec pétéchies, odeur fétide caractéristique, hémoglobinurie: }\end{array}$ & Guéri \\
\hline $\begin{array}{l}13 \\
14 \\
15 \\
16 \\
17 \\
18 \\
19\end{array}$ & $\begin{array}{c}13 \text { ans } \\
? \\
? \\
? \\
? \\
? \\
?\end{array}$ & $\begin{array}{l}13 \text { jours } \\
8 \text { jours } \\
8 \text { jours } \\
8 \text { jours } \\
8 \text { jours } \\
8 \text { jours } \\
13 \text { jours }\end{array}$ & $\begin{array}{l}\text { Tous ces animaux présentent la même symptomatologie : } \\
\text { Inappétence, abattement, congestion des muqueuses safranées, pas de } \\
\text { fièvrc. }\end{array}$ & $\begin{array}{l}\text { Guéri } \\
\text { Guéri } \\
\text { Guéri } \\
\text { Guéri } \\
\text { Guéri } \\
\text { Guéri } \\
\text { Guéri }\end{array}$ \\
\hline 20 & $?$ & 11 jours & $\begin{array}{l}\text { Inappétence, abattement, congestion des muqueuses safranées, pas de } \\
\text { fièvre, violentes crises nerveuses entraînant l'obligation de sacrifier } \\
\text { l'animal. }\end{array}$ & Mort \\
\hline $\begin{array}{l}21 \\
22 \\
23\end{array}$ & $\begin{array}{l}? \\
? \\
?\end{array}$ & $\begin{array}{l}11 \text { jours } \\
11 \text { jours } \\
11 \text { jours }\end{array}$ & $\begin{array}{l}\text { Inappétence, abattement, congestion des muqueuses safranées, pas de } \\
\text { fièvre. }\end{array}$ & $\begin{array}{l}\text { Guéri } \\
\text { Guéri } \\
\text { Guéri }\end{array}$ \\
\hline 24 & $?$ & 8 jours & $\begin{array}{l}\text { Inappétence, abattement, congestion des muqueuses safranées, pas de } \\
\text { fièvre, violentes crises nerveuses entrainant l'obligation d'abattre } \\
\text { l'animal. }\end{array}$ & Mort \\
\hline $\begin{array}{l}\text { de } 25 \\
\text { à } 32\end{array}$ & $?$ & 6 jours & $\begin{array}{l}\text { Inappétence, abattement, congestion des muqueuses safranées, pas de } \\
\text { fièvre. }\end{array}$ & $\left\{\begin{array}{c}\text { T'ous } \\
\text { Guéris }\end{array}\right.$ \\
\hline
\end{tabular}


L'étude histopathologique de la glande hépatique, organe toujours atteint, montre une congestion intense, une cytolyse des cellules hépatiques dans la zone centro-lobulaire, une dégénerescence graisseuse périlobulaire, la présence de pigments (hémosidérine) dans les espaces portes, une sclérose des espaces portes et de la capsule de Glisson.

En dehors de cette épidémie, plusieurs cas chez d'autres chevaux indigènes sont signalés mais ne peuvent être suivis, sauf un, dont nous parlerons brièvement.

Cheval, 10 ans, présenté à la clinique le 22 Avril avec.les commémoratifs suivants : inappétence, adynamisme, urine foncée. Température $36 \circ$, prostration, respiration lente et profonde, dos voussé; conjonctive safranée avec pétéchies, muqueuse buccale ictérique, salivation abondante, hémoglobinurie. Il meurt le lendemain.

Examen nécroptique : l'aspect général du sujet, après dépouillage, est jaune safran.

Cavité thoracique : léger œdème du poumon, spumosités à la coupe, cœur présentant une myocardite hypertrophiante.

Cavité abdominale et pelvienne : congestion de tous les viscères, hépatomégalie, splénomégalie, suffusions sanguines sur la muqueuse gastrique, meloena.

Vessie fortement distendue, hémoglobinurie.

Pyélite sérofibrineuse.

Le sang noir donne un caillot mal rétracté exsudant une petite quantité de sérum hémolysé.

Discussion. - Si les premiers cas et le dernier cités au cours de cette étude se rattachent dans les grandes lignes au tableau clinique de la HORSESICKINESS aiguë classique, e foyer du Ranch de l'Eperon en differe assez nettement par la fréquence des signes nerveux graves et l'apyrexie constante.

Il apparait d'ailleurs que, hors d'Afrique Occidentale Française, ces signes nerveux n'ont pas été, à notre connaissance, signalés. Et pourtant, dès 1896 , Pierre en fait état, de même Monfrais (1923). Et si Didier, dans sa thèse (1934) ne souligne pas cette particularité, pas plus qu'Advenier, dans la sienne récente (1946), Curasson, par contre, propose d'ajouter une forme nerveuse à la classification de Theiler (1930).

La classification de l'affection en diverses formes semble, en A.O.F., un peu artificielle, car la coexistence de symptômes variés est fréquente.

Nous ne sacrifierons pas au souci de systématisation didactique pour réclamer l'élargissement de la classification généralement admise par la création d'une forme norvcusc, complication, à notrc avis, de l'affection, plutôt qu'entité morbide.

En ce qui concerne la dénomination de cette sévère maladie, appelée successivement par les auteurs français, fière typhoilde bilieuse, fièvre pernicieuse, typho-malaria, typhus amaril équin, expressions imagees qui valent bien le nom actuel et passe-partout de peste équine, et tenant comple de la lésion hépatique constante. responsable, apparemment, de toutes les autres, on pourrait aussi bien admettre le terme d'ictère grave tropical infectieux du cheval, qui répond, sclon G. Roussy, pour l'ictère grave infectieux de l'homme, à une insuffisance fonctionnelle de la glande hépatique se traduisant par de l'ictère, des hémorragies, des troubles nerveux et un état adynamique prononcé entraînant la mort à plus ou moins brève échéance.

Au début de cet article, nous notons deux faits épidémiologiques particuliers à l'épizootie décrite :

a) l'affection n'évolue que chez les chevaux indigènes, de race locale, jouissant ordinairement d'une immunité partielle;

b) elle apparaît en pleine saison sèche et froide alors qu'il est devenu la règle de constater la maladie en fin de saison des pluies, d'Octobre à Décembre. Cette période de transition, qui précède la saison sèche, se caractérise par une chaleur et une humidité débilitantes et la présence constante d'insectes piqueurs, incriminés comme vecteurs de la maladie.

Conclusion. - Au cours d'une épizootie sévère de peste équine, ayant sévi à Dakar en 1942, nous avons souligné des faits épidémiologiques importants et inhabituels :

$1^{\circ}$ apparition de la maladie en saison sèche et froide, coïncidant avec la disparition des insectes piqueurs, vecteurs du virus;

$2^{\circ}$ atteinte grave et exclusive de chevaux de race locale, considérés généralement comme assez résistants,

Par ailleurs, nous insistons, du point de vue symptomatologique, sur la constance de l'apyrexie, la fréquence de l'hémoglobinurie, des troubles nerveux, dont les auteurs étrangers à l'A.O.F. ne font pas état.

$$
\text { (Laboratoire Central de l'Elevage, Dakar). }
$$

\section{BIDITOGRAPHIE}

HENNING (M.-W.). - Animal diseases in South Africa. 1932. DIDIER (L.). - Peste équime ox typhus amaril du cheval. Thèse Lyon, 1934.

CURASSON (G.). - Traité de pathologie exotique vétérinaire et comparée. 2 édition, T.I., 1942.

ADVENIER (C.). - Contubation à l'étude de la peste équine. These Alfort, 1946. 